BMJ Open Ophthalmology

\section{Use of four asymmetric marks to orient the donor graft during Descemet's membrane endothelial keratoplasty}

To cite: Matsuzawa A, Hayashi T, Oyakawa I, et al. Use of four asymmetric marks to orient the donor graft during Descemet's membrane endothelial keratoplasty. BMJ Open Ophth 2017;1:e000080. doi:10.1136/bmjophth-2017000080

Received 09 March 2017 Revised 16 May 2017 Accepted 27 June 2017

\section{CrossMark}

For numbered affiliations see end of article.

Correspondence to Dr Naoko Kato; naokato@bc. iij4u.or.jp

\section{ABSTRACT}

Introduction Maintaining the correct orientation of the donor graft is important during Descemet's membrane endothelial keratoplasty (DMEK). We describe a new method of marking the donor graft prior to DMEK.

Methods Twelve eyes of 10 patients with bullous keratopathy who underwent DMEK were retrospectively analysed. Donor discs were created by stripping the endothelium-Descemet's membrane layer from corneoscleral buttons. Four semicircular marks, two $1.0 \mathrm{~mm}$ and two $1.5 \mathrm{~mm}$ in diameter, were created at the edge of the donor disc. The small and large marks were paired. Each donor graft was inserted into the anterior chamber, unfolded and attached to the posterior corneal stroma with an air bubble.

Results The inserted grafts were all appropriately orientated when attached to the back surfaces of the corneas. The two pairs of asymmetric marks afforded valuable guidance. Even when the graft was partially folded or decentred, and one pair of marks was obscured, the other pair was always visible to indicate graft orientation. Best spectacle-corrected visual acuity improved significantly in all patients $(p<0.001)$.

Compared with the preoperative endothelial cell density of the donor graft, that of the corneal endothelium had decreased $44.0 \% \pm 10.0 \%$ by 6 months after surgery.

Conclusions Two pairs of asymmetrical semicircular marks placed on the edge of the donor graft allowed appropriate graft orientation during DMEK.

\section{INTRODUCTION}

Descemet's membrane endothelial keratoplasty (DMEK), first described by Melles et al in $2006,{ }^{1}{ }^{2}$ is one of the most useful forms of corneal transplantation when corneal endothelial decompensation is to be treated. In penetrating keratoplasty and Descemet-stripping automated endothelial keratoplasty, the transplanted tissues are either entire corneas or posterior lamellae of the corneal stroma together with the endothelia. In DMEK, however, the corneal graft is composed of only the corneal

\section{Key Messages}

What is already known about this subject?

D During Descemet's membrane endothelial keratoplasty, if the graft orientation is incorrect on attachment to the corneal stromal surface, primary graft failure may develop.

- Several investigators proposed their original methods to determine the correct orientation of the graft, such as the Moutsouris sign, S-stamp on the donor graft or asymmetric marks on the edge of the graft using a 1-millimetre-diameter dermatological biopsy punch.

What are the new findings?

- We described two pairs of small and large semicircular marks, using two $1.0 \mathrm{~mm}$ and two $1.5 \mathrm{~mm}$ in diameter at the edge of the donor disc.

How might these results change the focus of research or clinical practice?

Using this method, even when the graft was partially folded or when the graft was slightly decentred and one pair of marks became hidden, the other pair was visible, allowing correct graft orientation.

endothelium and Descemet's membrane. This means that irregularities on the anterior and posterior corneal surfaces are minimised, resulting in rapid improvement of visual acuity and low-level graft rejection. $^{3-5}$ Increasing numbers of ophthalmologists, particularly in the West, use DMEK to treat patients with corneal endothelial decompensation. ${ }^{6} 7$

However, DMEK requires high levels of skill and experience. It is critical to correctly identify graft orientation during surgery. The donor graft is only about $20 \mu \mathrm{m}$ thick and is rolled up with the endothelium on the exterior. Less experienced surgeons sometimes misidentify the graft orientation when the graft is expanded in the anterior 


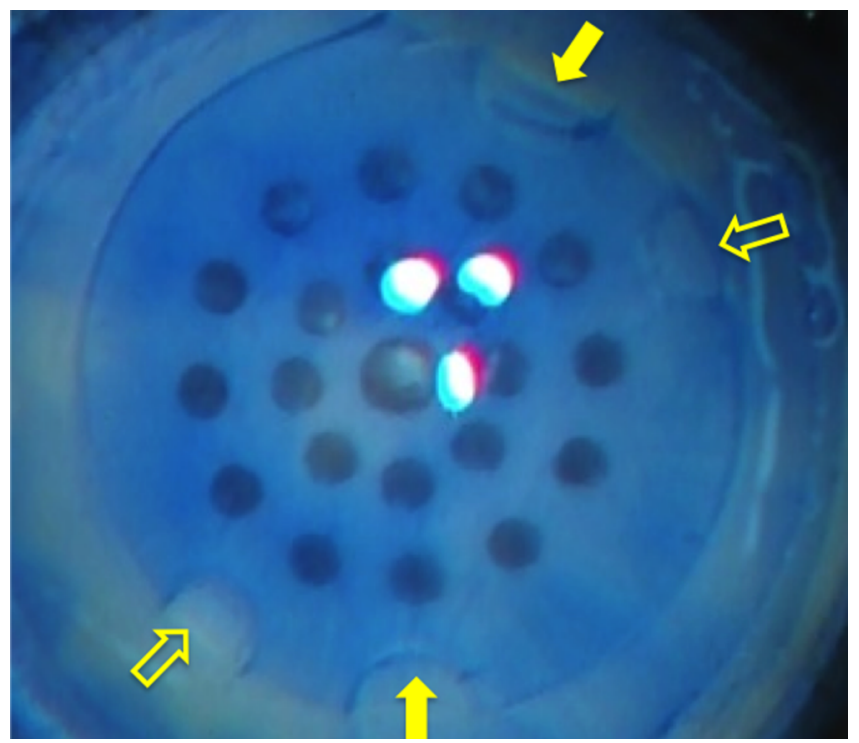

Figure 1 Photograph of a Descemet membrane graft in preparation. The operator uses a punch $7.5 \mathrm{~mm}$ in diameter. Four small marks are made, two of diameter $1.0 \mathrm{~mm}$ (open arrows) and the other two of diameter $1.5 \mathrm{~mm}$ (solid arrows). A 1.0millimetre-diameter and a 1.5-millimetre-diameter mark are paired on opposite sides of the graft.

chamber. If the graft orientation is incorrect on attachment to the corneal stromal surface (in other words, if the endothelium is appressed to that surface), primary graft failure may develop.

Several investigators have sought to avoid this complication. Dapena et al used the Moutsouris sign to determine the correct orientation of the graft. ${ }^{8}$ Veldman et al stamped an S on DMEK donor tissue to orientate the graft. ${ }^{9}$ Bachmann et al created asymmetric marks on the edge of the graft using a 1-millimetre-diameter dermatological biopsy punch. ${ }^{10}$ Bhogal et al had recently introduced single triangular mark using a $30^{\circ}$ incision knife. $^{11}$ Recently, several clinicians have employed intraoperative ocular coherence tomography (OCT) to determine donor graft orientation. ${ }^{12-14}$ All of these methods are effective, and we usually employ several of them during surgery.

We herein report a new marking method. We placed four asymmetric semicircular marks on the edge of the graft. These marks ensured correct graft orientation and will thus be of assistance to DMEK surgeons.

\section{METHODS}

\section{Patients}

Data on 12 eyes of 10 patients with bullous keratopathy who underwent DMEK at Yokohama Minami Kyosai Hospital from October 2015 to March 2016 and who were followed up for more than 6 months were retrospectively analysed. We treated three men and seven women with a mean age of $73.0 \pm 7.8$ years.

All eyes underwent DMEK alone. Four eyes had Fuchs' corneal endothelial dystrophy and three corneal endotheliopathy caused by pseudoexfoliation syndrome. Four eyes had iatrogenic bullous keratopathy; three had undergone argon laser iridotomy and one prior cataract surgery and intraocular lens implantation. One eye had a history of corneal endotheliitis, but there was no evidence of viral infection.

The study protocol was approved by the institutional review board of the Yokohama Minami Kyosai Hospital.

\section{DONOR PREPARATION}

We used precut donor tissue from SightLife (Seattle, WA, USA) for DMEK. Each graft was peeled as previously described. ${ }^{15}$ A punch was gently placed on the endothelial surface to indent a circle 7.5 or $7.75 \mathrm{~mm}$ in diameter. Next, dermatological biopsy punches (Kai Industries, Seki, Japan) 1.0 and $1.5 \mathrm{~mm}$ in diameter were used to place four small semicircular marks on the edge of the circle. The small and large marks were paired and the two pairs were at opposite ends of the graft diameter (figure 1). The marked donor graft was stained with trypan blue and stored in oxyglutathionecontaining intraocular irrigation solution until insertion 30-60 min later.

\section{Surgical techniques and postoperative treatment Procedure}

We performed all surgeries under local anaesthesia. After retrobulbar anaesthesia and establishment of a Nadbath facial nerve block, two small incisions and a 2.8millimetre-diameter upper corneal or corneoscleral incision were made. Peripheral iridectomywas performed at the 6-o'clock position using a 25-gauge vitreous cutter to prevent postoperative pupillary block. The prepared donor membrane graft was placed into an intraocular lens injector (WJ-60M; Santen Pharmaceuticals, Osaka, Japan) and inserted into the anterior chamber. A small amount of air was injected between the host cornea and 

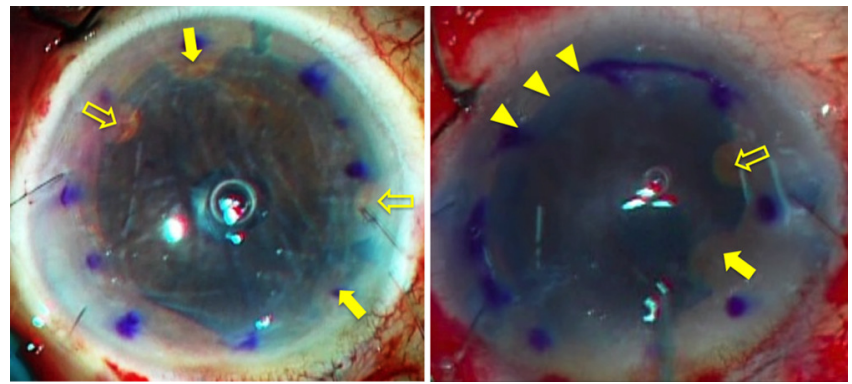

Figure 2 Intraoperative photographs of Descemet membrane grafts inserted into and unfolded within the anterior chamber. Left: the four small asymmetric markings can be clearly seen at the graft edge. Open arrows indicate the smaller marks (diameter $1.0 \mathrm{~mm}$ ) and solid arrows the larger marks (diameter $1.5 \mathrm{~mm}$ ). The smaller and larger marks are inversely located with respect to their orientation when at the time of their creation. Right: the inserted graft is partially folded at the edge (triangles), hiding one pair of the small and large marks. However, the other pair of marks is visible (open and solid arrows), showing that the graft is appropriately orientated.

the donor graft, and the rolled-up donor graft unfolded. The correct graft orientation was confirmed by reference to the marks (figure 2). The anterior chamber was filled with air to allow the graft to adhere to the host cornea. After $15 \mathrm{~min}$, the air was partially replaced with balanced salt solution. Finally, $0.4 \mathrm{mg}$ of betamethasone (Rinderon; Shionogi, Osaka, Japan) was subconjunctivally administered in $1.5 \%$ (w/v) levofloxacin eye drops (Cravit; Santen).

Postoperative medications included $1.5 \%(\mathrm{w} / \mathrm{v})$ levofloxacin (Cravit), $0.1 \%(\mathrm{w} / \mathrm{v})$ betamethasone sodium phosphate (Sanbetasone; Santen) and 2\% (w/v) rebamipide ophthalmic solution (Mucosta; Otsuka, Tokyo, Japan), commencing at four times daily for 3 months and tapering thereafter.

\section{Examinations}

In addition to the standard ophthalmic examination, the best spectacle-corrected visual acuity (BSCVA), corneal endothelial cell density (ECD), central corneal thickness and graft adaptation were evaluated preoperatively and for up to 6 months postoperatively. Graft adaptation was assessed by both slit-lamp biomicroscopy and anterior segment optical coherence tomography (SS1000; Tomey, Nagoya, Japan). Corneal thickness was measured by corneal tomography (SS1000; Tomey). The preoperative ECD was determined by reviewing eye bank donor records. Intraoperative and postoperative complications were recorded, and the postoperative ECD was measured using a specular microscope (FA3509; Konan Medical, Nishinomiya, Japan).

\section{Statistical analysis}

The Wilcoxon test was used to compare mean values, as appropriate. All analyses were performed using
StatView statistical software (Abacus Concepts). A $\mathrm{p}$ value of less than 0.05 was considered to reflect significance.

\section{RESULTS}

Both pairs of marks were readily recognisable during surgery (figure 2, left). Even when the graft was partially folded and one pair of marks was obscured, the other pair was visible, allowing correct graft orientation (figure 2, right). In addition, if the graft was slightly decentred and one pair of marks became hidden behind the chamber angle, the other pair was visible. Correct donor graft orientation was confirmed a few hours after surgery, using the marks, by slit-lamp biomicroscopy (figure 3).

No eye received a graft that was incorrectly orientated. The BSCVA (the logarithm of the minimum angle of resolution) improved significantly from $0.95 \pm 0.60$ preoperatively to $0.06 \pm 0.08$ at 6 months postoperatively $(p=0.001)$. The central corneal thickness decreased from $701.3 \pm 95.8 \mu \mathrm{m}$ preoperatively to $519.3 \pm 29.7 \mu \mathrm{m} 6$ months postoperatively $(\mathrm{p}<0.001)$. The corneal ECD was $1549.5 \pm 390.3$ cells $/ \mathrm{mm}^{2} \quad 6$ months postoperatively $(44.0 \% \pm 10.0 \%$ less than the preoperative value of the donor graft).

No eye showed any signs of pupillary block, microbial infection or endothelial rejection. Partial detachment of the graft, requiring re-bubbling of the anterior chamber, was observed in two eyes 4 days after surgery; both grafts became completely attached after re-bubbling. Notably, we encountered no case of primary graft failure.

\section{DISCUSSION}

We describe a new method whereby DMEK graft orientation can be intraoperatively assessed. We placed four small asymmetric marks on the edge of the graft. Graft orientation was easily determined both intraoperatively and postoperatively, and each graft were smoothly attached to the back surface of the cornea.

The use of a dermatology biopsy punch to create small marks is not entirely novel. Bachmann et al previously placed three asymmetric semicircles on the margin of donor grafts, ${ }^{10}$ achieving very successful results. However, when only three asymmetric marks are placed, graft orientation cannot be determined if the graft becomes dislocated and a part thereof is hidden behind the angle, or when the graft is partially folded at the edge. When two asymmetric pairs of marks are placed opposite each other on the graft edge, one pair is always visible when the other is obscured for various reasons. When the surgeon observes them during and after the surgery from the epithelial side, the large and small pair of marks is always observed in the opposite orders that were made during preparation with the endothelial side up. If the graft attached the incorrect orientation, the pair of marks can be recognised in the same order to that was observed during graft preparation. 


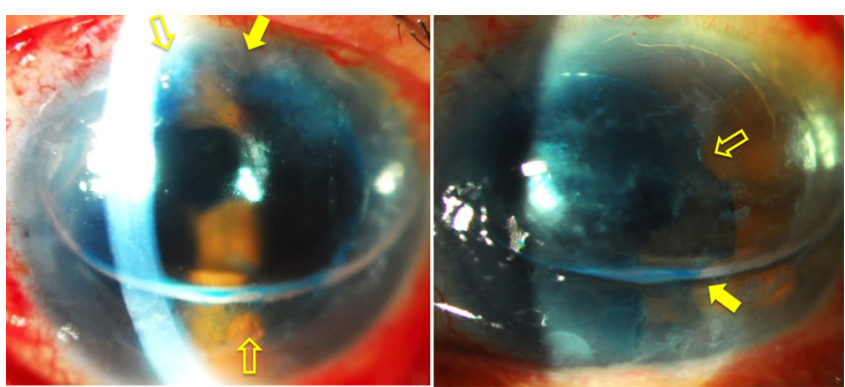

Figure 3 Slit-lamp photographs taken immediately after surgery. Left: both pairs of small (open arrows) and large (solid arrows) marks are visible, indicating that the graft is appropriately orientated. Right: although the graft is slightly decentred, and one pair of the marks (small and large) is hidden behind the chamber angle, the other pair (open and solid arrows) is visible.

A possible disadvantage of our method is that endothelial cells may be lost when the graft edge is punched. In the Bachmann's technique, three semicircular marks are made, using a $1 \mathrm{~mm}$ punch, on a donor graft $8 \mathrm{~mm}$ in diameter, associated with (theoretical) endothelial cell loss of $2.5 \% .^{10}$ Using our method, the theoretical loss is $5.8 \%$ (two semicircles $1.0 \mathrm{~mm}$ and two $1.5 \mathrm{~mm}$ in diameter in a 7.5-millimetre-diameter donor graft). If we used larger graft, such as $8.0,8.5$ or $9.0 \mathrm{~mm}$ in diameter, the theoretical cell loss decrease to $5.1 \%, 4.5 \%$ or $4.0 \%$, respectively. However, endothelial cell loss after DMEK ranges from $33.9 \%$ to $56 \%$ over the first 6 months; the small losses associated with graft marking are thus irrelevant. ${ }^{16}$ The loss of a few cells is preferable to incorrect graft positioning.

As surgeons gain experience, they become able to centre the graft without difficulty and to identify graft orientation easily. Also, novel endothelium-in techniques that may contribute to keep the endothelial cells dramatically have recently been introduced. ${ }^{18}{ }^{19}$ The two pairs of marks may then become unnecessary. However, it is easy to make the marks on opposite sides of the graft edge; this aids in graft manipulation during DMEK. We hope that our suggestion will help surgeons to ascend the DMEK learning curve.

\section{Author affiliations}

${ }^{1}$ Department of Ophthalmology, St. Marianna University School of Medicine, Kanagawa, Japan

${ }^{2}$ Department of Ophthalmology, Kawasaki Municipal Tama Hospital,

Kanagawa, Japan

${ }^{3}$ Department of Ophthalmology, Yokohama Minami Kyosai Hospital, Kanagawa, Japan

${ }^{4}$ Department of Ophthalmology, Yokohama City University School of Medicine, Kanagawa, Japan

${ }^{5}$ Department of Ophthalmology, Heart Life Hospital, Okinawa, Japan

${ }^{6}$ Department of Ophthalmology, Ryukyu University, Okinawa, Japan

${ }^{7}$ Department of Ophthalmology, Saitama Medical University, Saitama, Japan

Competing interests None declared.
Patient consent Obtained.

Provenance and peer review Not commissioned; externally peer reviewed.

Open Access This is an Open Access article distributed in accordance with the Creative Commons Attribution Non Commercial (CC BY-NC 4.0) license, which permits others to distribute, remix, adapt, build upon this work noncommercially, and license their derivative works on different terms, provided the original work is properly cited and the use is non-commercial. See: http:// creativecommons.org/licenses/by-nc/4.0/

(C) Article author(s) (or their employer(s) unless otherwise stated in the text of the article) 2017. All rights reserved. No commercial use is permitted unless otherwise expressly granted.

\section{REFERENCES}

1. Melles GR. Posterior lamellar keratoplasty: DLEK to DSEK to DMEK. Cornea 2006;25:879-81.

2. Melles GR, Ong TS, Ververs B, et al. Descemet membrane endothelial keratoplasty (DMEK). Cornea 2006;25:987-90.

3. Hayashi T, Yamagami S, Tanaka K, et al. Immunologic mechanisms of corneal allografts reconstituted from cultured allogeneic endothelial cells in an immune-privileged site. Invest Ophthalmol Vis Sci 2009;50:3151-8.

4. Anshu A, Price MO, Price FW. Risk of corneal transplant rejection significantly reduced with Descemet's membrane endothelial keratoplasty. Ophthalmology 2012;119:536-40.

5. Tourtas T, Laaser K, Bachmann BO, et al. Descemet membrane endothelial keratoplasty versus Descemet stripping automated endothelial keratoplasty. Am J Ophthalmol 2012;153:1082-90.

6. Ang M, Wilkins MR, Mehta JS, et al. Descemet membrane endothelial keratoplasty. $\mathrm{Br} J$ Ophthalmol 2016;100:15-21.

7. Dapena I, Ham L, Droutsas K, et al. Learning curve in Descemet's membrane endothelial keratoplasty: first series of 135 consecutive cases. Ophthalmology 2011;118:2147-54.

8. Dapena I, Moutsouris K, Droutsas K, et al. Standardized "no-touch" technique for Descemet membrane endothelial keratoplasty. Arch Ophthalmol 2011;129:88-94.

9. Veldman PB, Dye PK, Holiman JD, et al. Stamping an S on DMEK donor tissue to prevent upside-down grafts: laboratory validation and detailed preparation technique description. Cornea 2015;34:1175-8.

10. Bachmann BO, Laaser $\mathrm{K}$, Cursiefen $\mathrm{C}$, et al. A method to confirm correct orientation of Descemet membrane during Descemet membrane endothelial keratoplasty. Am J Ophthalmol 2010;149:922-5

11. Bhogal M, Maurino V, Allan BD. Use of a single peripheral triangular mark to ensure correct graft orientation in Descemet membrane endothelial keratoplasty. J Cataract Refract Surg 2015;41:2022-4.

12. Steven $\mathrm{P}$, Le Blanc $\mathrm{C}$, Velten $\mathrm{K}$, et al. Optimizing Descemet membrane endothelial keratoplasty using intraoperative optical coherence tomography. JAMA Ophthalmol 2013;131:1135-42.

13. Saad A, Sabatier P, Gatinel D. Graft orientation, optical coherence tomography, and endothelial keratoplasty. Ophthalmology 2013;120:871-871.e3.

14. Saad A, Guilbert E, Grise-Dulac A, et al. Intraoperative OCT-assisted DMEK: 14 consecutive cases. Cornea 2015;34:802-7.

15. Tenkman LR, Price FW, Price MO. Descemet membrane endothelial keratoplasty donor preparation: navigating challenges and improving efficiency. Cornea 2014;33:319-25.

16. Ham L, Dapena I, Liarakos VS, et al. Midterm results of Descemet membrane endothelial keratoplasty: 4 to 7 years clinical outcome. Am J Ophthalmol 2016;171:113-21.

17 Ang M, Mehta JS, Newman SD, et al. Descemet Membrane Endothelial Keratoplasty: Preliminary Results of a Donor Insertion Pull-through Technique Using a Donor Mat Device. Am J Ophthalmol 2016;171:27-34.

18. Parekh M, Ruzza A, Ferrari S, et al. Endothelium-in versus endothelium-out for Descemet membrane endothelial keratoplasty graft preparation and implantation. Acta Ophthalmol 2017;95:194-8.

19. Busin $M$, Leon $P$, Scorcia $V$, et al. Contact lens-assisted pull-through technique for delivery of tri-folded (endothelium in) DMEK grafts minimizes surgical time and cell loss. Ophthalmology 2016;123:476-83. 